\title{
Adaptación y validación de la Escala de Prejuicio Sutil y Manifiesto hacia inmigrantes venezolanos en una muestra colombiana
}

\author{
Jorge Palacio ${ }^{1}$, Ignacio Ramos-Vidal ${ }^{2}$, Humberto Llinas-Solano ${ }^{3}$, Andrea \\ Doria-Zapata ${ }^{4}$, Karen Nogueda-Cadena ${ }^{5}$ \\ Universidad del Norte - Colombia ${ }^{1,3,4}$, Universidad de Sevilla - España ${ }^{2}$, \\ Universidad de Puebla - México5
}

El objetivo del estudio fue establecer las propiedades psicométricas para Colombia de la Escala de Prejuicios Sutiles y Manifiestos elaborada por Pettigrew y Meertens (1995) y adaptada al español por Rueda y Navas (1996), tomando como población objetivo los inmigrantes venezolanos que han llegado a Colombia. La muestra fue de 1078 colombianos residentes en Barranquilla (Atlántico) seleccionados intencionalmente, con edades entre 18 y 78 años $(M=34.6$ y $D E=13.4)$. Un $49 \%(n=523)$ eran hombres. Los resultados indican una consistencia interna para la escala global de $\alpha=.84$ y $\omega=.85$. La subescala de Prejuicio Sutil muestra una consistencia interna de $\alpha=.74$ y $\omega=.75$, y la de Prejuicio Manifiesto de $\alpha=.78$ y $\omega=.80$. Con el Análisis Factorial Confirmatorio se ratifica la estructura factorial, pero se cambia el ítem 17 de factor y se elimina el ítem 7, quedando en total con 19 ítems. Se describen las limitaciones.

Palabras clave: prejuicio, racismo, validación, Colombia, inmigrantes, Venezuela.

\section{Adaptation and Validation of the Subtle and Blatant Prejudice Scale in a Colombian Sample}

The main objective of this study was to establish the psychometric properties for Colombia of the Subtle and Blatant Prejudice Scale of Pettigrew and Meertens (1995) which was

1 Doctor en Psicología. Profesor Departamento de Psicología. Universidad del Norte. Dirección postal: Km5 Antigua vía Puerto Colombia, Barranquilla, Colombia. Contacto: jpalacio@uninorte.edu.co. https://orcid.org/0000-0001-6971-7067

2 Doctor en Psicología. Profesor Departamento de Psicología Social. Universidad de Sevilla, España. Grupo de investigación CAVIDA Universidad Pontificia Bolivariana de Montería, Colombia. Dirección postal: Cra.6 No.97 A - 99, Colombia. Contacto: iramos5@us.es. https://orcid.org/0000-0002-1840-0761

3 Doctor en Estadística. Profesor Departamento Matemáticas y Estadística. Universidad del Norte. Dirección postal: Km5 Antigua vía Puerto Colombia, Barranquilla, Colombia. Contacto: hllinas@uninorte.edu.co https://orcid.org/0000-0002-2976-5109

4 Psicóloga. Sacyr construcción. Dirección postal: Carrera 10 C\#49-82 Barranquilla, Colombia. Contacto: andreadoria@uninorte.edu.co. https://orcid.org/0000-0003-1373-4698

5 Estudiante de la Facultad de Psicología. Universidad Autónoma de Puebla. Dirección postal: Calle 3 Ote. 1413, Barrio de Analco, CP 72500 Puebla, México. Contacto: cadenakaren. kn@gmail.com. https://orcid.org/0000-0003-3469-3151 
adapted to Spanish by Rueda and Navas (1996). Venezuelan immigrants in Colombia are the target population. The sample comprised 1078 Barranquilla residents (Atlantic) who were selected intentionally, with an age range between 18 and 78 years old $(M=34.6$ and $S D=13.4)$, of whom $49 \%(n=523)$ were male. Outcomes show a Cronbach's alpha $(\alpha)$ of .84 and an Omega $(\omega)$ of .85 for the global scale. The Subtle Prejudice subscale displays $\alpha=.74$ and $\omega=.75$, while the Blatant Prejudice subscale demonstrates $\alpha=.78$ and $\omega=.80$. Confirmatory Factor Analysis confirms the underlying factorial structure, but item 17 changes factor and item 7 is eliminated, leaving a total of 19 items. Limitations are discussed.

Keywords: Prejudice, racism, validation, Colombia, immigrants, Venezuela.

\section{Adaptaçáo e validaçáo da escala de preconceito sutil e flagrante em uma amostra colombiana}

O objetivo principal deste estudo foi estabelecer as propriedades psicométricas para a Colômbia da Escala de Preconceito Sutil e Flagrante de Pettigrew e Meertens (1995), adaptada ao espanhol por Rueda e Navas (1996), tendo como população alvo os imigrantes venezuelanos que chegaram à Colômbia. A amostra foi composta por 1078 moradores de Barranquilla (Atlántico) que foram selecionados intencionalmente, com uma faixa etária entre 18 e 78 anos $(M=34.6$ e $S D=13.4) .49 \%(\mathrm{n}=523)$ eram do sexo masculino. Os resultados mostram uma alta consistência interna geral $(\alpha=.84$ e $\omega=.85)$. Para a Escala de Preconceito Sutil $\alpha=.74$ y $\omega=.75$, e para a Escala de Preconceito flagrante $\alpha=.78$ y $\omega$ $=.80$. A Análise Fatorial Confirmatória ratifica a estrutura fatorial subjacente, mas o item 17 muda o fator e o item 7 é eliminado, deixando um total de 19 itens. As limitaçóes são discutidas.

Palavras-chave: preconceito, racismo, validação, Colômbia, imigrantes, Venezuela.

\section{Adaptation et validation de l'échelle des préjugés subtils et flagrants à leégard des immigrants vénézuéliens dans un échantillon colombien}

L'objectif principal de cette étude était d'établir les propriétés psychométriques pour la Colombie de l'Échelle des préjugés subtils et manifestes de Pettigrew et Meertens (1995), adaptée en espagnol par Rueda et Navas (1996), en prenant comme population cible les immigrants vénézuéliens arrivés en Colombie. L'échantillon était composé de 1078 résidents de Barranquilla (Atlántico) qui ont été sélectionnés intentionnellement, avec une tranche d'âge comprise entre 18 et 78 ans $(M=34.6$ y $S D=13.4) .49 \%(\mathrm{n}=523)$ étaient des hommes. Les résultats montrent un alpha $(\alpha)$ de .84 et une Omega $(\omega)$ de .85 pour les deux échelles au sens large. Pour la sous échelle Prejudice Sutil, une $\alpha=, 74$ et $\omega=, 75$, et pour la sous échelle Prejudice Manifeste $\alpha=.78$ et $\omega=.80$. L'analyse factorielle confirmatoire ratifie la structure factorielle sous-jacente, mais l'item 17 est changé de facteur, et l'item 7 est éliminé, laissant un total de 19 items. Les limitations sont discutées.

Mots-clés: préjugés, racisme, validation, Colombie, immigrants, Venezuela. 
El prejuicio es definido por Allport (1954) como un sentimiento de antipatía basado en una generalización inflexible y errónea que puede ser expresada hacia un grupo o un individuo, por ser miembro de un determinado grupo. Como esta aproximación involucra tanto aspectos afectivos como cognitivos, se puede asumir que el prejuicio sostiene ideologías que pueden justificar conductas discriminatorias hacia determinados grupos (Pettigrew \& Meertens, 1995). Al prejuicio se le han adjudicado funciones asociadas con la jerarquización, con el proceso de categorización social, y también se ha utilizado para comprender la hostilidad hacia grupos marginales (Cárdenas, Music, Contreras, Yeomans \& Calderon, 2007). Sin embargo, en cuanto a las percepciones o manifestaciones hostiles hacia los miembros del exogrupo, diferentes propuestas muestran que el prejuicio tradicional no captura todas las actitudes prejuiciosas — que resultan cada vez más sofisticadas - debido a que los sujetos tratan de evitar posibles sanciones sociales derivadas de su exhibición pública.

En este sentido, Pettigrew y Meertens (1995) diferencian dos tipos de prejuicios en situaciones de contacto intergrupal: el prejuicio sutil y el prejuicio manifiesto. El prejuicio manifiesto es la forma tradicional de rechazo, es decir aquella que resulta evidente y fácil de identificar, mientras que el prejuicio sutil se relaciona con formas latentes de discriminación y puede resultar más difícil de percibir en situaciones de interacción social (Meertens \& Pettigrew, 1997; Swim, Ferguson \& Hyers, 1999). El prejuicio manifiesto se ha caracterizado por una combinación de rechazo formal y una falta de disposición para mantener relaciones personalizadas con miembros del exogrupo (Meertens \& Pettigrew, 1997). No obstante, las expresiones directas de estas actitudes no son socialmente aceptadas, y es allí donde emerge el prejuicio sutil, que resulta más difícil de detectar que el manifiesto porque se focaliza en conductas discriminatorias indirectas y encubiertas (Krolikowski, Rinella \& Ratcliff, 2015). 
Para la evaluación de estas formas de prejuicio, Pettigrew y Meertens (1995) diseñaron la Escala de Prejuicio Manifiesto y Sutil que estaba compuesta por 20 ítems, que originalmente tenía como objetivo analizar la percepción de los europeos hacia los migrantes que llegaban de la extinta Unión Soviética y de las subsecuentes revoluciones en los países de Europa Oriental. La muestra original fue de 3810 europeos mayores de 18 años, de siete países y en cuatro idiomas - francés, holandés, inglés y alemán. La escala de obtuvo una consistencia interna y validez - de constructo y de criterio- adecuadas para la subescala manifiesta (el índice alfa de Cronbach oscilaba entre .87 y .90) y sutil (oscilaba entre .73 y .82).

La subescala de prejuicio manifiesto se estructura sobre los factores de (a) Amenaza y rechazo del exogrupo, dado que los percibe como un factor de riesgo para la supervivencia del endogrupo; y (b) Rechazo a la intimidad o a un contacto íntimo con miembros del exogrupo (de carácter sexual y sentimental). La escala de prejuicio sutil se estructura sobre los factores de (a) la Defensa de los valores tradicionales, bajo la creencia que los valores endogrupales son más relevantes que los del exogrupo; (b) la Exageración de las diferencias culturales, que está dada por la percepción negativa de las creencias y prácticas religiosas del exogrupo con respecto al endogrupo, asumiendo que existe una brecha cultural amplia; y (c) la Negación de las emociones positivas, o en otras palabras, la supresión de sentimientos positivos hacia el exogrupo (p.ej. solidaridad y admiración).

Por otro lado, la escala trata de diferenciar los niveles de prejuicio en una población determinada, mediante la elaboración de una tipología de tres perfiles de prejuicio al cruzar las dos subescalas: (1) El primer perfil, denominado "igualitario" describe sujetos con niveles de prejuicio bajos en las dos subescalas; (2) El segundo perfil, denominado "fanático" agrupa sujetos con niveles elevados de prejuicio para ambas subescalas; (3) el tercer perfil, llamado "sutil", agrupa sujetos con altos niveles en la subescala sutil y bajos en la manifiesta, y (4) un cuarto grupo de sujetos con un nivel sutil bajo y un nivel manifiesto alto quedan en el perfil de "error", que indica una apreciación que es 
ambigua o no coherente entre sí. Es generalmente un grupo minoritario (no más del 6\% de la muestra).

La escala originalmente en inglés, se adaptó y validó al español por Rueda y Navas (1996), con una muestra de 160 estudiantes españoles entre 15 y 35 años. A ellos se les evaluó los prejuicios sobre tres colectivos minoritarios distintos - gitanos, magrebíes y negros. A nivel global la escala presentó una fiabilidad óptima $(\alpha=.84)$, y para la escala de prejuicio manifiesto y sutil se identificó un índice de fiabilidad aceptable ( $\alpha=.75$ y .74, respectivamente). La consistencia interna por población fue la siguiente: Gitanos: Prejuicio Manifiesto .78; Prejuicio Sutil .79; Magrebíes: Prejuicio Manifiesto .76, Prejuicio Sutil .68; Negros: Prejuicio Manifiesto .70 y Prejuicio Sutil de 68 .

En Latinoamérica se adaptó y validó la escala en Costa Rica por Smith, Araya y Peña (2006). El estudio se conformó con una muestra de 278 costarricenses entre los 17 y 65 ańos; a un 49\% de la muestra se les preguntó sobre migrantes nicaragüenses y el otro $51 \%$ sobre migrantes colombianos. Se obtuvo una consistencia interna de .77 para el prejuicio manifiesto, con un coeficiente de discriminación mayor de .40. Para el prejuicio sutil se obtuvo un alfa de .73 con un coeficiente de discriminación mayor de .30. En esta validación se omitió el ítem con respecto a la disposición a intimar con otro grupo étnico puesto que en estudios anteriores mostraron dificultades de comprensión asociadas a él.

Otra adaptación se llevó a cabo en Chile por Cárdenas et al. (2007). La muestra fue de 324 participantes entre los 16 y los 21 ańos. Respondían con respecto a inmigrantes bolivianos y grupos étnicos minoritarios de Chile. El alfa de la subescala de prejuicio manifiesto fue de .73, y de prejuicio sutil de .68. La estructura factorial de la subescala de prejuicio manifiesto se mantuvo estable, pero para el prejuicio sutil, los ítems se agruparon solo en dos factores, fusionando los factores en Diferencias Culturales y Valores Tradicionales, en lugar de los tres factores del estudio original.

Recientemente se realizó una validación en Argentina por Müller, Ungaretti y Etchezahar (2017), donde el grupo de comparación eran 
los "villeros", adjetivo que suele emplearse para denominar a personas que residen en áreas periurbanas de bajos recursos. Se tomó una muestra de 266 residentes en Buenos Aires con edades entre 19 y 55 años. La consistencia interna para la escala global fue de .84 , para el prejuicio manifiesto de .72. y para el prejuicio sutil $\alpha=.68$.

\section{Una aproximación al contexto migratorio colombiano}

Si bien Colombia era considerado un país con poca recepción de inmigrantes, en los últimos ańos alrededor de 1.4 millones de venezolanos han ingresado en territorio colombiano con tres objetivos: (1) transitar a otro país; (2) establecerse en las zonas fronterizas esperando que la crisis de Venezuela llegue a su fin; y (3) establecerse de forma permanente (Migración Colombia, 2019, CODHES, 2018). Estas condiciones propician el asentamiento de ciudadanos venezolanos en diferentes ciudades, que independientemente de su estatus legal, se encuentran la mayoría de ellos, en situaciones de exclusión y vulnerabilidad (ONU, 2018; CODHES, 2018, Fernández-Nińo, Luna-Orozco, Navarro-Lechuga, Flórez-García, Acosta-Reyes, Solano, Bravo \& Goenaga, 2018; Schwartz, Salas-Wright, Pérez-Gómez, Mejía-Trujillo, Brown, Montero-Zamora, et al., 2018).

En el Departamento del Atlántico, situado en el noroeste de Colombia, se estima que ha llegado un $8.2 \%$ de los inmigrantes venezolanos que han entrado a Colombia en el último año (Migración Colombia, 2018). La mayor parte se ha asentado en municipios periféricos a la capital, y se han identificado al menos tres asentamientos en Barranquilla, en los cuales se ha documentado la emergencia de conflictos entre la población local y los recién llegados (Blanquicet, 2019). La aparición de conductas discriminatorias y conflictos intergrupales suele venir precedida de diferentes tipos de prejuicios que van condicionando progresivamente las relaciones intergrupales. Un estudio reciente muestra que la población local, si bien tiene una actitud de apoyo, empieza a percibir a los recién llegados como una amenaza en tanto son observados como competidores por los escasos recursos 
comunitarios y de ayudas de diferente tipo ofrecidas por el gobierno en estos barrios vulnerables (Martinez-González, Palacio, Romero \& Rodelo, 2018).

Debido a los antecedentes expuestos, el objetivo de este estudio es adaptar la Escala de Prejuicio Sutil y Manifiesto [Diseñada originalmente por Pettigrew y Meertens (1995) y posteriormente adaptada y validada al castellano por Rueda y Navas (1996)], y revisar sus propiedades psicométricas para determinar si dicho instrumento es capaz de discriminar actitudes prejuiciosas de la población local con respecto a los inmigrantes venezolanos. Se espera hacer un seguimiento al nivel de prejuicio a medida que se incrementen las relaciones inter-grupales con un amplio potencial de conflictividad social entre la población local y el colectivo de migrantes venezolanos que han llegado a esta región del país.

\section{Método}

\section{Participantes}

La muestra de esta investigación está conformada por 1078 colombianos que residen en la ciudad de Barranquilla, con edades comprendidas entre 18 y 78 años $(M=34.4 ; D E=13.4)$. Un $49 \%$ de los participantes son hombres. La muestra fue seleccionada mediante un muestreo no probabilístico intencional, con la condición de inclusión de edad, nacionalidad y tiempo de vivir en la ciudad - mayores de edad, colombianos y que habiten de manera continua en Barranquilla hace más de 17 años. Para las características socioeconómicas, el $11.1 \%$ de la población se encuentra en estrato 1 (el más vulnerables), el 17.6\% en estrato 2; el 38\% en estrato 3; el $19.1 \%$ en estrato 4 , el $5.8 \%$ en estrato 5 y el $8.1 \%$ en estrato 6 (el de mayor solvencia económica). Con respecto al nivel educativo, un $25 \%$ de la muestra terminó los estudios de secundaria, un $22 \%$ tiene estudios técnicos o de formación profesional, y un $34.2 \%$ tiene estudios universitarios. 


\section{Medición}

Se empleó la Escala de Prejuicios Sutiles y Manifiestos diseñada por Pettigrew y Meertens (1995) en la versión posteriormente adaptada y validada por Rueda y Navas (1996), siguiendo la puntuación de la escala original. Está compuesta por 20 ítems que se promediaban de una puntuación de 1 a 5 (ej. 1 = Totalmente en desacuerdo a 5 =Totalmente de acuerdo). El ítem 18 se puntúa en una escala de molestia -indiferencia, y el 19 y 20 en frecuencia - de nunca a muy frecuente-ente. Los ítems se reparten en las dos subescalas con cinco factores en total (Ver tabla 1):

\section{Tabla 1}

Items de prejuicio por subescalas y factores

\begin{tabular}{lll}
\hline Subescala & Factores & Ítems \\
\hline Sutil & Valores tradicionales (VT) & $1,3,5,6,14$ \\
& Diferencias culturales (DC) & $11,12,16$ \\
& Emociones positivas (EP) & 19,20 \\
\hline \multirow{2}{*}{ Manifiesta } & Amenaza y rechazo (AR) & $2,8,9,10,15,17$ \\
& Intimidad (I) & $4,7,13,18$ \\
\hline
\end{tabular}

Además, según lo propuesto por Pettigrew y Meertens (1995) para comprobar la validez de las subescalas, se indagó sobre la Percepción de las políticas migratorias, por lo cual se hicieron dos preguntas: (1) ¿Considera usted que los derechos de los inmigrantes indocumentados deberían ser restringidos, mantenerse igual o ser extendidos? (2) En su opinión, ¿qué politica debería adoptar el pais con respecto a los inmigrantes venezolanos? Se daban varias opciones que iban desde opciones radicales (p.ej., deportar a todos los inmigrantes, o deportar a los venezolanos no nacidos en Colombia) hasta las más flexibles (p.ej., deportar a los venezolanos que no tengan los documentos en regla, o no deportarlos). 


\section{Procedimiento}

El cuestionario fue adaptado al contexto de estudio para lo cual se realizó un pilotaje con 15 sujetos, lo cual permitió controlar el tiempo y anticipar dificultades de comprensión, así como propiciar el entrenamiento de un grupo de estudiantes universitarios. Las recomendaciones de los participantes se introdujeron en la versión final del instrumento, las cuales se referían a organizar las opciones de respuesta de las características sociodemográficas. El estudio fue aprobado por el comité de ética de la Universidad y luego, con el apoyo de los estudiantes, se aplicó a los sujetos quienes firmaron un consentimiento informado de manera voluntaria, y respondieron el cuestionario de forma anónima.

\section{Análisis de datos}

El procesamiento de los datos se realizó a través del software libre R. (RProject, 2019). En primer lugar, se realizó un análisis factorial exploratorio de cada una de las dos subescalas que componen el instrumento para determinar su índice de fiabilidad. Posteriormente, se les realizó un análisis factorial confirmatorio. Los indicadores de validez se evidenciaron a través de la discriminación de la tipología de prejuicio con los dos ítems que apuntaban a los Derechos de los inmigrantes y a la Política de Inmigración Futura, tal como se realizó en el estudio de Pettigrew y Meertens (1995).

\section{Resultados}

\section{Análisis factorial exploratorio para la Subescala de Prejuicio Sutil}

Con el fin de aplicar este método, se comprobaron los supuestos básicos del análisis:

1) Multicolinealidad entre variables: Se cumple la prueba de esfericidad de Bartlett $\left(\chi^{2}=199.12, \mathrm{df}=45, p<.00001\right)$, por lo que se 
rechazó la hipótesis nula que la matriz de correlaciones es igual a la matriz identidad $(p<.05)$.

2) Prueba KMO de colinealidad (Kaiser, 1970): se determinó que hay un nivel suficiente de multicolinealidad entre las variables ( $\mathrm{KMO}=.772$ considerado bueno).

Los 10 ítems de la escala sutil forman una estructura factorial exactamente igual a la obtenida en estudios anteriores (Pettigrew \& Meertens, 1995; Cárdenas et al. 2007; Müller, et al., 2017). Es decir que los ítems se agrupan en los 3 factores (mediante rotación oblicua) y se observa que las cargas más altas se distribuyen en los factores teóricos esperados. En total, el porcentaje de varianza explicada por los tres factores es del 36.5\% (Ver tabla 2).

\section{Tabla 2}

Distribución factorial de la Subescala de Prejuicio Sutil

\begin{tabular}{|c|c|c|c|c|c|}
\hline Factores & Ítems & $\begin{array}{c}\text { Carga en } \\
\text { VT* }^{*}\end{array}$ & $\begin{array}{c}\text { Carga en } \\
\text { DC }^{* *}\end{array}$ & $\begin{array}{c}\text { Carga en } \\
\text { EP*** }^{* *}\end{array}$ & Comunalidad \\
\hline \multirow{5}{*}{$\begin{array}{l}\text { Valores } \\
\text { tradicionales } \\
(\mathrm{VT})^{*}\end{array}$} & 1 & .49 & -.09 & .09 & .26 \\
\hline & 3 & .59 & .07 & -.05 & .36 \\
\hline & 5 & .45 & .16 & .15 & .25 \\
\hline & 6 & .37 & .12 & -.36 & .28 \\
\hline & 14 & .40 & -.04 & -.05 & .16 \\
\hline \multirow{3}{*}{$\begin{array}{l}\text { Diferencias } \\
\text { culturales } \\
(\mathrm{DC})^{* *}\end{array}$} & 11 & .20 & .60 & -.02 & .40 \\
\hline & 12 & -.04 & .86 & -.02 & .74 \\
\hline & 16 & .05 & .43 & .26 & .26 \\
\hline \multirow{2}{*}{$\begin{array}{l}\text { Emociones } \\
\text { positivas } \\
(\mathrm{EP})^{* * *}\end{array}$} & 19 & -.05 & -.09 & .42 & .19 \\
\hline & 20 & .02 & .01 & .87 & .76 \\
\hline \multicolumn{2}{|c|}{$\begin{array}{l}\text { Porcentaje de varianza } \\
\text { explicada }\end{array}$} & $11.3 \%$ & $13.5 \%$ & $11.7 \%$ & Total $=36.5 \%$ \\
\hline
\end{tabular}




\section{Análisis factorial exploratorio para la Subescala de Prejuicio Manifiesto}

Se aplicó análisis factorial exploratorio para encontrar los factores subyacentes en los 10 ítems de la subescala de prejuicio manifiesto. Se utilizaron matrices de correlaciones policóricas por tratarse de escalas tipo Likert ordinales. El resultado indica que se satisfacen los supuestos básicos para poder aplicar el análisis, ya que se cumple la prueba de esfericidad de Bartlett $\left(\chi^{2}=207.856, \mathrm{df}=45, p<.00001\right), \mathrm{y}$ el $\mathrm{KMO}=.762$, lo cual es considerado como un valor bueno. Es decir, podemos considerar que hay un nivel suficiente de multicolinealidad entre las variables (Ver tabla 3).

Si bien la estructura factorial es similar a los resultados de otras validaciones, se observa, por un lado, una diferencia en el ítem 17 (Los venezolanos proceden de razas menos capaces y esto explica por qué viven en una situación peor que la de los colombianos), el cual se encuentra en el factor de Intimidad en lugar del factor de Amenaza y rechazo como está en el estudio original. Por otro lado, el ítem 7 (No me importaría tener relaciones sexuales con una persona venezolana) aparece en el Factor de Amenaza y Rechazo y no en el de Intimidad como lo plantean estudios anteriores (Smith et al., 2006; Cárdenas et al. 2007; Müller et al., 2017). Aun así, el aporte de este ítem a ambos factores es similar dado que sus cargas factoriales no difieren entre sí, a diferencia del ítem 17 cuyo peso en el Factor Intimidad es de .44 y el de Amenaza y Rechazo es de .35. Debido a que el peso del ítem 7 es bajo, se sugiere eliminarlo en esta versión (Ver tabla 3), tal como hicieron los autores del estudio de validación desarrollado con población costarricense (Smith et al., 2006). 


\section{Tabla 3}

Distribución factorial Subescala de Prejuicio Manifiesto

\begin{tabular}{lcccc}
\hline Factores & Ítems & Carga en AR* $^{*}$ & Carga en ${ }^{* *}$ & Comunalidad \\
\hline Amenaza y & 2 & .45 & -.08 & .21 \\
rechazo (AR)* & 8 & .48 & .36 & .36 \\
& 9 & .58 & -.11 & .35 \\
& 10 & .49 & .31 & .34 \\
& 15 & .54 & -.23 & .34 \\
& $7^{* * *}$ & -.23 & .15 & .08 \\
\hline Intimidad (I)** & $17^{* * * *}$ & .35 & .44 & .32 \\
& 4 & -.04 & -.52 & .27 \\
& 13 & -.03 & -.69 & .48 \\
& 18 & -.08 & .85 & .73 \\
\hline $\begin{array}{l}\text { Porcentaje de varianza } \\
\text { explicada }\end{array}$ & $14.9 \%$ & $19.8 \%$ & Total $=34.7 \%$ \\
\hline
\end{tabular}

*** Ítem para excluir. ${ }^{* * * *}$ Pasa del factor AR al I.

\section{Análisis de fiabilidad}

Se evaluó la consistencia interna de cada una de las escalas a través del estadístico alfa de Cronbach $(\alpha)$ (Welch \& Comer, 1988), indicador que evalúa en qué medida están correlacionados los ítems que forman parte de una misma escala. Algunos autores, (Lord \& Novick, 1968; Cortina, 1993; Yang \& Green, 2011), señalan que su uso debe hacerse con precaución siendo preciso tener en consideración los supuestos de normalidad, errores correlacionados y tau-equivalencia (es decir, cargas factoriales iguales en todos los ítems). Por esta razón, la consistencia también se analizó con el índice Omega $(\omega)$ desarrollado por McDonald (1999). Según Trizano-Hermosilla y Alvarado (2016), este coeficiente es útil cuando hay equivalencia (en este caso, omega y alfa coinciden matemáticamente), y cuando hay factores con cargas diferentes. 
Para el análisis de confiabilidad de las escalas se utilizaron tres estructuras factoriales diferentes para comparar los diferentes omegas y alfas obtenidos según: (1) La estructura factorial conforme lo propone Pettigrew y Meertens (1995); (2) La estructura factorial encontrada en este artículo, sin eliminar ítems; (3) La estructura factorial eliminando ítems.

En la estructura 1 se encontró que globalmente la consistencia interna - el Alfa de Cronbach $(\alpha)$ tiene un valor de .84 y un Omega $(\omega)$ de .85. Para la escala de Prejuicio Sutil, el alfa es de .74 y el omega .75. Para los factores que la componen: Defensa de los valores tradicionales el alfa es de .65 y el omega .66; Exageración de las diferencias culturales con un alfa de .71 y omega .74; Emociones Positivas con alfa .61 y omega .61. Por otro lado, para la escala de Prejuicio Manifiesto, el alfa es de .76 y el omega .78, que es mayor al de la escala de prejuicio sutil. Para el factor de Amenaza y Rechazo el alfa fue de .74, y el omega de .75, y para Intimidad el alfa fue de .62, y el omega de .70 .

Para la estructura 2, se encontró que globalmente el instrumento tiene un $\alpha=.84$ al igual que en la situación anterior. Para la escala de Prejuicio Sutil y los factores que la componen, el resultado es idéntico. No obstante, para la escala de Prejuicio Manifiesto se encontró que tiene un alfa .76 y omega .78; para los factores que la componen los datos son los siguientes: Amenaza y Rechazo con alfa .66 y omega .68 e Intimidad con alfa .82 y omega .82 .

En la estructura 3, como se mencionó anteriormente, el ítem 7 aparece con una carga baja en ambos factores de la misma escala de Prejuicio Manifiesto, y el ítem 17 aparece con el mismo peso en ambos factores. En la tabla 4 se muestra los resultados generales del análisis de confiabilidad sin el ítem 7, lo cual va en favor de quitarlo de esta versión, y el ítem 17 dejarlo en el factor de Intimidad. 


\section{Tabla 4}

Análisis de Confiabilidad de las Subescalas de Prejuicio Sutil y Manifiesto sin el item 7

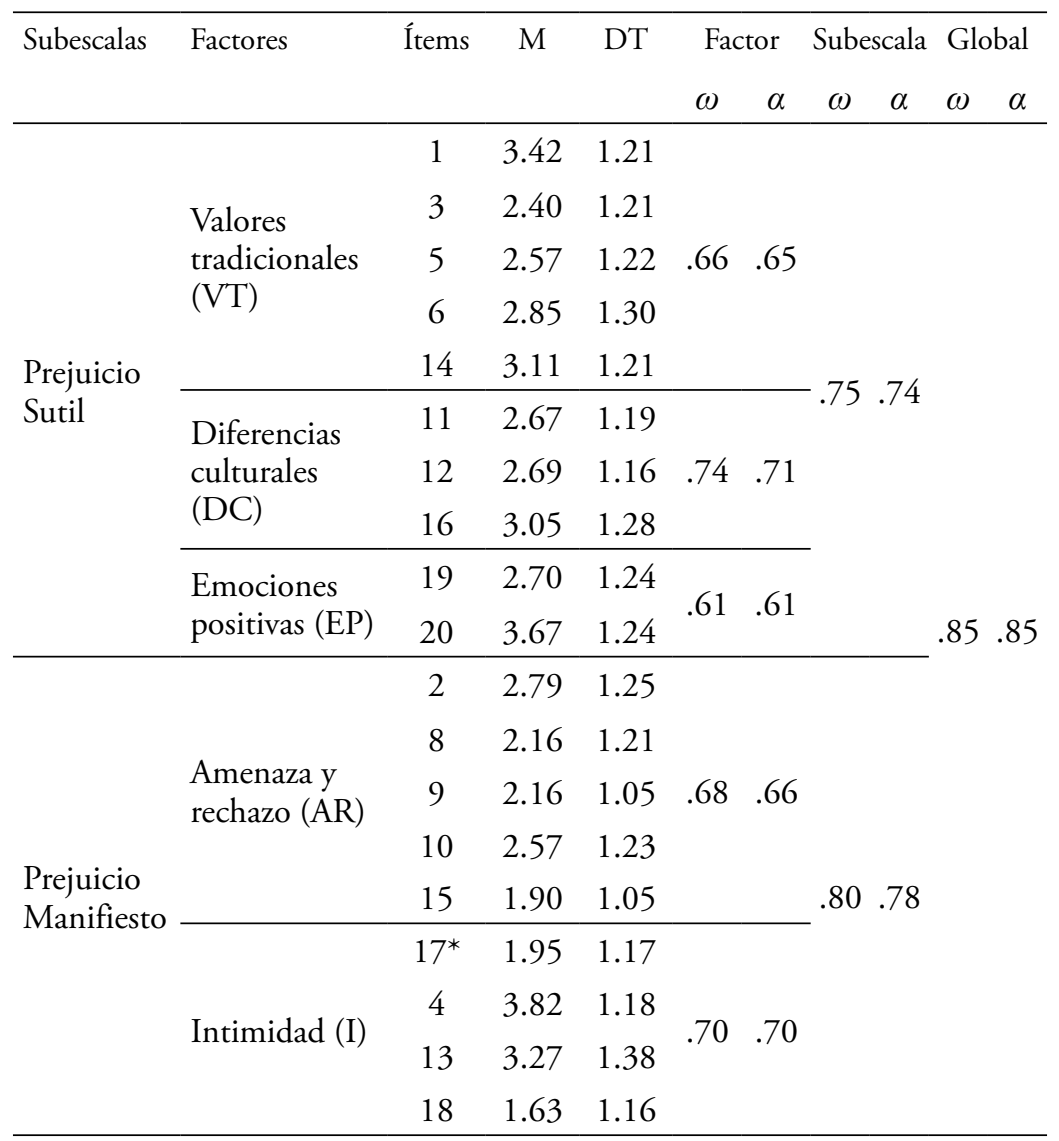

Fuente: Elaboración propia. ${ }^{*}$ Pasa de AR al I.

\section{Análisis factorial confirmatorio}

Para ejecutar este procedimiento, las métricas de las variables latentes se estandarizaron y se utilizó la matriz de correlaciones en lugar de la de covarianzas para estimar los parámetros del modelo. Siguiendo 
los lineamientos de Hu y Bentler (1999), la bondad del ajuste se evaluó con los índices de Comparative Fit Index (CFI), el Root Mean Square Error of Approximation (RMSEA) y el Standardized Root Mean Squeare Residual (SRMR). De acuerdo a los indicadores, el modelo es aceptable ya que los índices se aproximan a los rangos sugeridos. Para muestras mayores o iguales a 250 sujetos, algunos autores (Hu \& Bentler, 1999; $\mathrm{Yu}, 2002)$ han propuesto que se tiene un ajuste aceptable cuando el CFI está entre .80 y .95 , y tanto el RMSEA, como el RMSR, están entre .05 y .08 (Ver tabla 5).

\section{Tabla 5}

Indicadores del Modelo Factorial Confirmatorio de las Subescalas de Prejuicio Sutil y Manifiesto

\begin{tabular}{|c|c|c|c|c|c|c|}
\hline Estructura factorial & chisq & $\mathrm{df}$ & $p$ & $\mathrm{CFI}^{*}$ & RMSEA $^{* *}$ & $\mathrm{SRMR}^{* * *}$ \\
\hline $\begin{array}{l}\text { 1. Estructura } \\
\text { teórica (Pettigrew \& } \\
\text { Meertens, 1995) }\end{array}$ & 1455.11 & 159 & $<.00001$ & .78 & .09 & .09 \\
\hline $\begin{array}{l}\text { 2. Estructura } \\
\text { encontrada sin } \\
\text { eliminar ítems }\end{array}$ & 1412.29 & 159 & $<.00001$ & .79 & .09 & .09 \\
\hline $\begin{array}{l}\text { 3. Estructura } \\
\text { eliminando ítem } 7\end{array}$ & 1274.54 & 141 & $<.00001$ & .80 & .09 & .09 \\
\hline
\end{tabular}

Fuente: Elaboración propia.

${ }^{*}$ Comparative Fit Index. ${ }^{* *}$ Root Mean Square Error of Approximation. ${ }^{* *}$ Standardized Root Mean Squeare Residual.

\section{Indicadores de Validez}

Para la validez se procedió a construir la tipología de prejuicio propuesta por Pettigrew y Meertens (1995). Se dividieron las puntuaciones de las subescalas en altas y bajas tomando el 3 como punto medio de corte. De tal forma que aquellos puntajes superiores a 3 en ambas escalas son considerados altos, y los puntajes inferiores a 3 son considerados bajos. Al hacer el cruce se crean las tipologías de prejuicio de toda la muestra (Ver tabla 6). 


\section{Tabla 6}

Distribución de los tipos de prejuicio

\begin{tabular}{lcc}
\hline Tipos de prejuicio & Total & Porcentaje (\%) \\
\hline Igualitarios & 555 & 51.5 \\
Sutiles & 367 & 34.0 \\
Fanáticos & 81 & 7.5 \\
Error & 74 & 6.8 \\
\hline Total & 1077 & 100 \\
\hline
\end{tabular}

Fuente: Elaboración propia.

Como se indica en la tabla 6 , los porcentajes de cada grupo son similares a estudios anteriores, siendo mayor el grupo de Igualitarios donde se ubican aquellos con puntuaciones inferiores a 3 en las dos subescalas. Sin embargo, el porcentaje del grupo de Error es un poco mayor a lo esperado. Luego se procedió a utilizar la tipología como factor de segmentación con la primera pregunta sobre los derechos de los inmigrantes para realizar la medida de validez adicional. En la tabla 7 se observa el cruce con respecto a los derechos de los inmigrantes.

Los resultados obtenidos son similares a los de estudios anteriores (Rueda y Navas, 1996; Cárdenas et al., 2007). En general existe una predisposición de los Igualitarios a ampliar o mejorar los derechos de los inmigrantes venezolanos. Para los sutiles se observan tres grupos diferenciados, un porcentaje repartido de $35 \%$ considera que deben ser restringidos o mantenerlos igual, y un $28.6 \%$ considera que deben ser ampliados. Mientras que en el grupo de Fanáticos se observa una fuerte tendencia - 50.6\% - para restringir los derechos de los inmigrantes (Ver tabla 7). 
Adaptación y validación de la Escala de Prejuicio Sutil y Manifiesto / Palacio et al.

\section{Tabla 7}

Opiniones sobre los derechos de los inmigrantes y tipología de prejuicio

Pregunta

Tipología de prejuicio (\%)

\begin{tabular}{lllll}
\hline Considera usted que & (1) Restringidos & 24.5 & 35.4 & $\mathbf{5 0 . 6}$ \\
$\begin{array}{l}\text { los derechos de los } \\
\text { inmigrantes venezolanos }\end{array}$ & (2) Mantenerse igual & 36.9 & $\mathbf{3 5 . 9}$ & 32.0 \\
\cline { 2 - 5 } deben ser & (3) Ampliados & $\mathbf{3 8 . 5}$ & 28.6 & 17.2 \\
\hline
\end{tabular}

Fuente: Elaboración propia.

En la segunda pregunta relacionada con la política futura que debe adoptar el país con respecto a los inmigrantes, se observa en los tres grupos una tendencia marcada a elegir la opción 4 de «Deportar a aquellos que han cometido delitos» (Ver tabla 8).

\section{Tabla 8}

Opiniones sobre politica futura del pais frente a los inmigrantes y tipología de prejuicio

\section{Pregunta}

Tipología de prejuicio (\%)

Igualitario $\quad$ Sutil $\quad$ Fanático

\begin{tabular}{|c|c|c|c|c|}
\hline \multirow{6}{*}{$\begin{array}{l}\text { ¿Qué política } \\
\text { debería } \\
\text { adoptar el } \\
\text { país con } \\
\text { respecto a los } \\
\text { inmigrantes } \\
\text { venezolanos? }\end{array}$} & $\begin{array}{l}\text { (1) Deportar a todos los } \\
\text { inmigrantes venezolanos }\end{array}$ & 1.8 & 1.9 & 19.7 \\
\hline & $\begin{array}{l}\text { (2) Deportar a los venezolanos no } \\
\text { nacidos en Colombia }\end{array}$ & 0.3 & 1.9 & 6.1 \\
\hline & $\begin{array}{l}\text { (3) Deportar a los venezolanos que } \\
\text { no contribuyen con la economía }\end{array}$ & 6.1 & 6.3 & 9.8 \\
\hline & $\begin{array}{l}\text { (4) Deportar a aquellos que han } \\
\text { cometido delitos }\end{array}$ & 68.9 & 71.6 & 46.9 \\
\hline & $\begin{array}{l}\text { (5) Deportar a aquellos que no } \\
\text { tengan los papeles en regla }\end{array}$ & 10.0 & 12.9 & 4.9 \\
\hline & (6) No deportarlos & 12.7 & 5.2 & 4.9 \\
\hline
\end{tabular}

Fuente: Elaboración propia. 
Si bien, un 68\% del grupo igualitario se ubica en esta opción, un $10 \%$ sostiene que deben deportar a aquellos que no tengan los papeles en regla y un $12.7 \%$ afirma que deberían No deportarlos. De los Sutiles, un $71.6 \%$ respondió Deportar a aquellos inmigrantes que han cometido delitos y un $12.9 \%$ opina que deberían deportar a aquellos que no tengan los papeles en regla. De los fanáticos, un $46 \%$ afirma que deben deportar a aquellos inmigrantes que han cometido delitos y un 19\% opina que deben deportarlos a todos. Fuera de la tendencia mayoritaria, se observa que los igualitarios tienen una tendencia a una política más suave que los sutiles y estos a su vez más moderada que los fanáticos.

\section{Discusión}

El propósito del estudio fue adaptar y validar la Escala de Prejuicio Sutil y Manifiesto desarrollada por Pettigrew y Meertens (1995) con una muestra colombiana hacia la población inmigrante venezolana. El instrumento ha sido validado previamente con índices de confiabilidad e indicadores de validez adecuados en otros países de Latinoamérica y España (Rueda y Navas, 1996; Smith et al., 2006; Cárdenas et al., 2007; Muller, Ungurietta \& Etchezahar, 2017).

El análisis factorial mostró una distribución similar a la del estudio original (Pettigrew \& Meertens, 1995) y a la de posteriores validaciones (Cardenas et al., 2007; Muller et al., 2017), con una ligera variación en la ubicación del ítem 17, el cual, en lugar de encontrarse en el factor de Amenaza y Rechazo, se ubicó en el factor Intimidad. Se decidió eliminar el ítem 7 (No me importaría tener relaciones sexuales con una persona venezolana), dado que el peso para el factor Amenaza y Rechazo es relativamente bajo y no se encontró en el factor Intimidad, como originalmente se estipuló por los autores de la escala. Además, se ha eliminado por motivos de comprensión y factores contextuales asociados con la sensibilidad de los participantes, tal como sucedió en la validación costarricense (Smith et al., 2006). 
Con el análisis factorial confirmatorio, se obtuvo un valor significativo en el índice de ajuste comparativo en la tercera situación donde se elimina el ítem 7 de la escala, y que valida su estructura factorial. Los valores de los Alfa de Cronbach con la nueva estructura factorial son adecuados (Escala Global .85; Escala de Prejuicio Manifiesto .78; Escala de Prejuicio Sutil .74), y se comportan de manera similar a los de otras validaciones con población latinoamericana (Smith et al., 2006; Cárdenas et al., 2007; Müller et al., 2017). Asimismo, se mantiene la tendencia que la consistencia interna de la subescala de prejuicio manifiesto es mayor a la de prejuicio sutil, lo cual puede deberse a la naturaleza del constructo. Pettigrew y Meertens (1995) sostienen que el prejuicio manifiesto es de tipo directo y más fácilmente observable mientras que estudios sobre el prejuicio sutil $-\mathrm{o}$ también denominado prejuicio moderno- sugieren que es ambivalente $y$, por tanto, más difícil de detectar debido a que expresa la negatividad de forma encubierta, a través de la negación de la discriminación, o al colocar condiciones para la aceptación de miembros de grupos minoritarios (Krolikowski et al., 2015; Anderson, 2017).

Los indicadores de validez indican que la escala permite discriminar las modalidades de prejuicios (ver tabla 6), y en el ítem sobre los derechos de los inmigrantes venezolanos indocumentados existe una tendencia de los igualitarios a ampliarlos, los sutiles se distribuyen entre ampliarlos y mantenerlos igual, y los fanáticos a restringirlos. Asimismo, con respecto a las políticas futuras que debe implementar el país con respecto a los inmigrantes venezolanos, los igualitarios muestran una tendencia hacia políticas flexibles (deportar a los delincuentes y no deportarlos) y los fanáticos una tendencia hacia políticas menos flexibles y más radicales (deportar a los delincuentes o deportarlos a todos) lo que es consistente con resultados anteriores (Cárdenas et al., 2017).

Otro punto para resaltar es el gran número de personas con perfil Igualitario (51.9\%), que puede sugerir un efecto de deseabilidad social de ambas escalas (i.e., King \& Bruner, 2000), en parte debido a que los ítems de las dos subescalas apuntan a comportamientos concretos, o bien o por el mismo carácter ambivalente del prejuicio sutil (Anderson, 2017). 


\section{Conclusión}

El prejuicio es un fenómeno de carácter psicosocial cuyo estudio sistemático permite comprender el proceso y resultado que describe las relaciones intergrupales (Allport, 1954). Sin embargo, la evaluación de este fenómeno se torna compleja debido a la dificultad de observar las diferentes modalidades que el prejuicio puede adoptar, desde formas fácilmente observables como la desvalorización pública de los miembros del exogrupo (i.e., prejuicio manifiesto) hasta fenómenos difíciles de percibir como las actitudes paternalistas hacia el exogrupo (i.e., prejuicio sutil), que con frecuencia se asocian a una percepción de dominancia del endogrupo hacia el exogrupo. La naturaleza dual del prejuicio exige por un lado evaluar de forma simultánea las distintas formas que puede adoptar este fenómeno, y por otro aconseja refinar, validar y adaptar contextualmente los instrumentos de medición para que pueden captar con mayor fidelidad la complejidad inherente a este fenómeno con amplio potencial para condicionar las relaciones intergrupales.

En esta investigación los indicadores de validación mostraron que la escala discrimina la tipología de prejuicios (ver tabla 6). Se han probado las hipótesis de estudio, donde en el ítem sobre los derechos de los inmigrantes venezolanos indocumentados muestra la tendencia de los igualitarios a ampliarlos, los sutiles se distribuyen entre ampliarlos y mantenerlos igual, y los fanáticos a restringirlos. Asimismo, con respecto a las políticas futuras que debe implementar el país con respecto a los inmigrantes venezolanos, los igualitarios muestran una tendencia hacia políticas flexibles y los fanáticos una tendencia hacia políticas menos flexibles y radicales, resultados similares a los encontrados en estudios previos (Cárdenas et al., 2017). Por lo anterior, se puede afirmar que la Escala de Prejuicio Sutil y Manifiesto desarrollada originalmente por Pettigrew y Meertens (1995) es válida para evaluar ambos tipos de prejuicios en población colombiana hacia el colectivo de inmigrantes venezolanos. Es necesario desarrollar futuros estudios que permitan identificar aquellos factores que activan los prejuicios hacia los colectivos minoritarios en general, y hacia el colectivo de migrantes 
venezolanos en particular, en aras a diseñar políticas públicas capaces de reducir los prejuicios que a su vez pueden conducir hacia la discriminación y los conflictos intergrupales generando una disrupción en la dinámica social.

\section{Limitaciones}

Una de las limitaciones de la investigación es que el estudio se llevó a cabo con una muestra colombiana residente en Barranquilla, por lo que es recomendable desarrollar un estudio con un muestreo representativo de áreas urbanas y rurales en el país. Además, se debe tener en consideración la homogeneidad de la muestra en variables como el estrato socioeconómico y el nivel educativo. Se sugiere, también, explorar variables como la ocupación y la religión dada su relevancia en temas relacionados con el desarrollo de percepciones negativas hacia los inmigrantes (Scheve \& Slaughter, 2001; Sahver, Troughton, Sibley \& Bulbulia, 2016).

De igual modo es necesario replicar la investigación en otras regiones del país debido a la amplia diversidad cultural que caracteriza a Colombia y a la densidad diferenciada de inmigrantes en cada región (i.e. Fearon, 2003, p. 218). Además, se sugiere realizar el estudio apuntando a diferentes colectivos de inmigrantes y no solamente con inmigrantes venezolanos, tal como lo han hecho en otros estudios (Rueda \& Navas, 1996; Smith et al., 2006; Cárdenas et al., 2007; Müller et al., 2017).

\section{Referencias}

Allport, G.W. (1954). The nature of prejudice. Massachusetts: AddisonWesley Publishing Company.

Anderson, J. (2017). The Prejudice against Asylum Seeker Scale: Presenting the Psychometric Properties of a new measure of classical and conditional Attitudes. Journal of Social Psychology, 158(6), 694-710. https://doi.org/10.1080/00224545.2017.1404958 
Blanquicet, J. (2019). En Colombia, 2.729 venezolanos han solicitado asilo. El Heraldo. Recuperado de: https:/www.elheraldo.co/ barranquilla/en-colombia-2729-venezolanos-han-solicitadoasilo-643496

Cárdenas, M., Music, A., Contreras, P., Yeomans, H. \& Calderón, C. (2007). Las nuevas formas de prejuicio y sus instrumentos de medida. Revista de Psicología, 16(1), 69-96. https://doi.org/10.5 354/0719-0581.2007.18435

CODHES (2018). Necesidades de protección de las personas venezolanas forzadas a migrar, refugiadas y en riesgo de apatriada en Colombia. Recuperado de: https://codhes.files.wordpress.com/2018/07/ informe-migrantes-y-refugiados-vz-en-col.pdf

Cortina, J. (1993). What is coefficient alpha? An examination of theory and applications. J. Appl. Psychol., 78, 98-104. https:// doi.org/10.1037/0021-9010.78.1.98

Domínguez Lara, S.A. (2014). ¿Matrices policóricas/ tetracoricas o matrices Pearson? Un estudio metodológico. Revista Argentina de Ciencias del Comportamiento, 6(1), 39-48.

Fearon, J. D. (2003). Ethnic and cultural diversity by country. Journal of Economic Growth, 8(2), 195-222. https://doi.org/ 10.1023/A:1024419522867

Fernández-Niño JA, Luna-Orozco K, Navarro-Lechuga E, FlórezGarcía V, Acosta-Reyes J, Solano A, et al. (2018). Necesidades percibidas de salud por los migrantes desde Venezuela en el asentamiento de Villa Caracas - Barranquilla, 2018: reporte de caso en salud pública. Rev Univ Ind Santander Salud, 50(3), 269-276. https://doi.org/10.18273/revsal.v50n3-2018002

Freiberg Hoffman, A., Stover, J., De la Iglesia, G. \& Fernández Liporace, M. (2013). Correlaciones policóricas y tetracóricas en estudios factoriales exploratorios y confirmatorios. Ciencias Psicologicas, 7(2), 151-161.

Hu, L. \& Bentler, P. M. (1999). Cutoff criteria for fit indexes in covariance structure analysis: conventional criteria versus new 
alternatives. Structural Equation Modeling, 6, 1-55. https://doi. org/10.1080/10705519909540118

Kaiser, H. F. (1970). A second generation little jiffy. Psychometrika, 35(4), 401-415. https://doi.org/10.1007/BF02291817

King, M. F. \& Bruner, G. C. (2000). Social desirability bias: A neglected aspect of validity testing. Psychology \& Marketing, 17(2), 79-103. https://doi.org/10.1002/(SICI)1520-6793(200 002) $17: 2<79:: A I D-M A R 2>3.0 . C O ; 2-0$

Krolikowski, A., Rinella, M. \& Ratcliff, J. (2015). The influence of the expression of subtle and batlant sexual prejudice on personal prejudice and identification with the expresser. Journal of Homosexuality. https://doi.org/10.1080/00918369.2015.10 83776

Lloret-Segura, S. Ferreres-Traver, A., Hernández-Baeza, A. y TomásMarco, I. (2014). El análisis factorial exploratorio de los ítems: una guía práctica, revisada y actualizada. Anales de Psicología, 30(3), 1151-1169. https://doi.org/10.6018/analesps.30.3.199361

Lord, F.M. \& Novick, M.R. (1968). Statistical Theories of Mental Test Scores. Menlo Park, CA: Addison-Wesley Publishing Company. Martinez-Gonzalez, Palacio, J., Romero, F. \& Rodelo, G. (2018). Acculturation patterns of venezuelan inmigrants in BarranquillaColombia. Conference IACCP: Acculturation patterns of venezuelan inmigrants in Barranquilla - Colombia at July 2018. https://doi.org.10.13140/RG.2.2.21945.47202

Meredith, W. (1993). Measurement invariance, factor analysis and factorial invariance. Psychometrika, 58(4), 525-543. https://doi. org/10.1007/BF02294825

Migración Colombia (2019). En Colombia ya hay más de 1.4 millones de venezolanos. Recuperado de: https://migravenezuela.com/web/ articulo/cuantos-venezolanos-hay-en-colombia-hasta-hoy/1390 Migración Colombia (2018). Infografía PEP Colombia. Recuperado de: http://migracioncolombia.gov.co/index.php/es/prensa/infografias/ categorias-migratorias/7921-infografia-pep 
Müller, M., Ungaretti, J. \& Etchezahara, E. (2017). Validación argentina de la Escala de Prejuicio Sutil y Manifiesto hacia villeros. Revista de Psicologia, 26(1), 1-13.

McDonald, R. (1999). Test Theory: a Unified Treatment. Mahwah, NJ: Lawrence Erlbaum Associates.

Organización Internacional de las Migraciones (2018). Informe sobre las migraciones en el mundo. Recuperado de https://publications. iom.int/system/files/pdf/wmr_2018_sp.pdf

Pettigrew, T. F. \& Meertens, R.W. (1995). Subtle and Blatant prejudice in Western Europe. European Journal of Social Psychology, 25, 57-75. https://doi.org/10.1002/ejsp.2420250106

Rueda, J. F. y Navas, M. (1996). Hacia una evaluación de las nuevas formas del prejuicio racial: las actitudes sutiles del racismo. Revista de Psicología Social, 11(2), 131-149. https://doi. org/10.1174/02134749660569314

Scheve, K.J. \& Slaugther, M.J. (2001). Labor Market Competition and Individual Preferences over Immigration Policy. The Review of Economics and Statistics, 83(1), 133-145. https://doi.org/ 10.1162/003465301750160108

Schwartz, S., Salas-Wright, C., Pérez-Gómez, A., Mejía-Trujillo, J., Brown, E., Montero-Zamora, P., Mecad, A., Scaramuttia, C., Soaresa, M., Vosa, S., Javakhishvilie, N., Dickson-Gomez, J. (2018). Cultural stress and psychological symptoms in recent Venezuelan inmigrants to the United States and Colombia. International Journal of Intercultural Relations, 67, 25-34. https://doi. org/10.1016/j.ijintrel.2018.09.001

Shaver, J.H., Troughton, G., Sibley, C.G. \& Bulbulia, J.A. (2016). Religion and the Unmaking of Prejudice toward Muslims: Evidence from a Large National Sample. PLoS ONE, 11(3) https:// doi.org/10.1371/journal.pone.0150209

Smith, V., Araya, M.A. y Peña, L. (2006). Representaciones de la diversidad cultural y actitudes hacia colectivos étnicos minoritarios e inmigrantes. Informe Final de Investigaciones Psicológicas. Universidad de Costa Rica: San José, Costa Rica. 
Trizano-Hermosilla, I. \& Alvarado, J. M. (2016). Best Alternatives to Cronbach's Alpha Reliability in Realistic Conditions: Congeneric and Asymmetrical Measurements. Frontiers in Psychology, 7(34). https://doi.org/10.3389/fpsyg.2016.00769

Welch, S. \& Comer, J. C. (1988). Quantitative methods for public administration: Techniques and applications. Chicago, IL: Dorsey Press.

Yang, Y. \& Green, S.B. (2011). Coefficient Alpha: a reliability coefficient for the 21st Century? J. Psychoeduc. Assess., 29, 377-392. https://doi.org/10.1177/0734282911406668

Yu, C. Y. (2002). Evaluating cutoff criteria of model fit indices for latent variable models with binary and continuous outcomes. Tesis doctoral. (No publicada). Recuperado el 3 de octubre de 2018, de http://www.statmodel.com/download/Yudissertation.pdf

Recibido: 12 de diciembre, 2018

Revisado: 26 de agosto, 2019 Aceptado: 11 de setiembre, 2019 
Anexo 1

Escala de Prejuicios Sutiles y Manifiestos (Pettigrew \& Meertens, 1995), adaptada para Colombia sobre migrantes venezolanos

Lea cuidadosamente y señale con una $X$ la opción que mejor represente su opinión de acuerdo a las siguientes afirmaciones utilizando la siguiente escala.

\begin{tabular}{|c|c|c|c|c|c|}
\hline & $\begin{array}{l}\text { Totalmente } \\
\text { en } \\
\text { desacuerdo }\end{array}$ & $\begin{array}{c}\text { En } \\
\text { desacuerd } \\
\text { o }\end{array}$ & $\begin{array}{l}\text { Ni en acuerdo } \\
\text { ni en } \\
\text { desacuerdo }\end{array}$ & $\begin{array}{c}\text { De } \\
\text { acuerdo }\end{array}$ & $\begin{array}{l}\text { Totalmente } \\
\text { de acuerdo }\end{array}$ \\
\hline $\begin{array}{l}\text { 1. En Colombia existen grupos que salen adelante por sus propios } \\
\text { esfuerzos. Los inmigrantes venezolanos deberían hacer lo mismo sin que } \\
\text { se les tenga que dar un trato especial. }\end{array}$ & 1 & 2 & 3 & 4 & 5 \\
\hline $\begin{array}{l}\text { 2. La mayoría de políticos en Colombia se preocupa mucho por los } \\
\text { venezolanos y no lo suficiente por los ciudadanos colombianos. }\end{array}$ & 1 & 2 & 3 & 4 & 5 \\
\hline $\begin{array}{l}\text { 3. El hecho de que los venezolanos no salgan adelante, es porque } \\
\text { enseñan a sus hijos valores y destrezas que no son las adecuadas en esta } \\
\text { sociedad. }\end{array}$ & 1 & 2 & 3 & 4 & 5 \\
\hline $\begin{array}{l}\text { 4. No me importaría si un inmigrante venezolano adecuadamente } \\
\text { preparado fuera profesor o jefe mío. }\end{array}$ & 1 & 2 & 3 & 4 & 5 \\
\hline $\begin{array}{l}\text { 5. El inconveniente de que los venezolanos se introduzcan en } \\
\text { determinados lugares (apartamentos, casas, lugares públicos, etc.) es que } \\
\text { no saben respetar las normas de convivencia establecidas. }\end{array}$ & 1 & 2 & 3 & 4 & 5 \\
\hline $\begin{array}{l}\text { 6. Los venezolanos se diferencian mucho de los colombianos en los } \\
\text { hábitos de higiene y en la necesidad de limpieza. }\end{array}$ & 1 & 2 & 3 & 4 & 5 \\
\hline $\begin{array}{l}\text { 7. Los colombianos y los venezolanos nunca pueden estar realmente } \\
\text { cómodo entre sí, incluso sin son amigos cercanos. }\end{array}$ & 1 & 2 & 3 & 4 & 5 \\
\hline $\begin{array}{l}\text { 8. La mayoría de los inmigrantes venezolanos que recibe algún tipo de } \\
\text { ayuda económica no lo necesitan, y podrían vivir sin ella si quisieran. }\end{array}$ & 1 & 2 & 3 & 4 & 5 \\
\hline $\begin{array}{l}\text { 9. Los venezolanos ocupan trabajos, viviendas y puestos escolares que no } \\
\text { saben utilizar y que deberían ser ocupados por otras personas. }\end{array}$ & 1 & 2 & 3 & 4 & 5 \\
\hline $\begin{array}{l}\text { 10. Por lo que conozco, las personas venezolanas son muy diferentes de } \\
\text { los colombianos en los valores que enseńan a sus hijos. }\end{array}$ & 1 & 2 & 3 & 4 & 5 \\
\hline $\begin{array}{l}\text { 11. Pienso que los inmigrantes venezolanos son muy diferentes al } \\
\text { colombiano, en sus ideas y valores sexuales o en su práctica sexual. }\end{array}$ & 1 & 2 & 3 & 4 & 5 \\
\hline $\begin{array}{l}\text { 12. No me importaría que un inmigrante venezolano, con una situación } \\
\text { económica parecida a la mía, se casara con alguien de mi entorno } \\
\text { familiar. }\end{array}$ & 1 & 2 & 3 & 4 & 5 \\
\hline $\begin{array}{l}\text { 13. Lo cierto es que si los venezolanos se esforzaran más podrían estar, al } \\
\text { menos, tan cómodamente como los ciudadanos colombianos. }\end{array}$ & 1 & 2 & 3 & 4 & 5 \\
\hline $\begin{array}{l}\text { 14. No puede uno confiar en los venezolanos pues ellos no conocen la } \\
\text { honestidad. }\end{array}$ & 1 & 2 & 3 & 4 & 5 \\
\hline $\begin{array}{l}\text { 15. Por lo que he podido ver, los inmigrantes venezolanos son muy } \\
\text { diferentes de los colombianos en su forma de hablar y de comunicarse } \\
\text { con la gente. }\end{array}$ & 1 & 2 & 3 & 4 & 5 \\
\hline $\begin{array}{l}\text { 16. Los venezolanos proceden de razas menos capaces y esto explica por } \\
\text { qué viven en una situación peor que la de los colombianos. }\end{array}$ & 1 & 2 & 3 & 4 & 5 \\
\hline \multirow[t]{2}{*}{$\begin{array}{l}\text { 17. Suponga usted que un hijo(a) suyo tiene un hijo(a) con una persona } \\
\text { venezolana. ¿Qué tanto le molestaría si su nieto(a) tuviese todos los } \\
\text { rasgos físicos de esa persona venezolana? }\end{array}$} & $\begin{array}{c}1 \\
\text { No le } \\
\text { molestaría } \\
\text { para nada }\end{array}$ & $\begin{array}{c}2 \\
\text { Le } \\
\text { molestarí } \\
\text { a un poco }\end{array}$ & $\begin{array}{c}3 \\
\text { Le es } \\
\text { Indiferente }\end{array}$ & $\begin{array}{c}4 \\
\text { Le } \\
\text { molestarí } \\
\text { a }\end{array}$ & $\begin{array}{c}5 \\
\text { Estaría muy } \\
\text { molesto }\end{array}$ \\
\hline & Nunca & $\begin{array}{l}\text { Muy } \\
\text { Pocas } \\
\text { Veces } \\
\end{array}$ & Algunas veces & $\begin{array}{l}\text { Frecuente } \\
\text { mente }\end{array}$ & $\begin{array}{l}\text { Muy } \\
\text { frecuentem } \\
\text { ente }\end{array}$ \\
\hline 18. ¿Has sentido admiración alguna vez por inmigrantes venezolanos? & 1 & 2 & 3 & 4 & 5 \\
\hline $\begin{array}{l}\text { 19. ¿Con qué frecuencia has sentido compasión por la situación en la } \\
\text { que se encuentran los inmigrantes venezolanos? }\end{array}$ & 1 & 2 & 3 & 4 & 5 \\
\hline
\end{tabular}

